\title{
Características cefalométricas dos indivíduos Padrão I
}

\author{
Sílvia Augusta Braga Reis*, Leopoldino Capelozza Filho **, Maurício de Almeida Cardoso***, \\ Marco Antônio Scanavini ${ }^{\star \star \star \star}$
}

\begin{abstract}
Resumo
As características cefalométricas de uma amostra de 30 pacientes brasileiros, adultos, leucodermas, Padrão I, selecionados a partir da avaliação morfológica de fotografias do perfil, foram estudadas em telerradiografias laterais com o objetivo de definir um padrão de referência, considerando-se as médias e, principalmente, os desvios padrões para estudos comparativos com amostras portadoras de discrepâncias esqueléticas. $\mathrm{Na}$ avaliação do padrão de crescimento facial obteve-se $9,4^{\circ} \pm 3,2^{\circ}$ para o ângulo do plano palatino e $121,4^{\circ} \pm 5,3^{\circ}$ para o ângulo goníaco, ambos apresentando dimorfismo sexual. $\mathrm{O}$ ângulo do plano mandibular apresentou média de $29,2^{\circ} \pm 4,2^{\circ}$, sem diferença entre os gêneros. Os valores obtidos para as alturas faciais total, inferior, média e posterior foram, respectivamente, $123,0 \mathrm{~mm} \pm 8,3 \mathrm{~mm} ; 68,8 \mathrm{~mm} \pm 6,6 \mathrm{~mm}$; $55,9 \mathrm{~mm} \pm 3,5 \mathrm{~mm}$ e $62,6 \mathrm{~mm} \pm 4,7 \mathrm{~mm}$. Todas essas variáveis foram significativamente maiores no gênero masculino. Os valores das medidas que definiram a relação maxilo-mandibular corroboraram o equilíbrio esquelético da amostra, pois observou-se $82,2^{\circ} \pm 2,9^{\circ}$ para o SNA; $79,8^{\circ} \pm 2,5^{\circ}$ para o $\mathrm{SNB}$ e $2,4^{\circ} \pm 1,4^{\circ}$ para o ANB, sem dimorfismo sexual. Os valores obtidos para os comprimentos efetivos da maxila e da mandíbula foram $95,2 \mathrm{~mm} \pm 5,7 \mathrm{~mm}$ e $124,2 \mathrm{~mm}$ $\pm 8,2 \mathrm{~mm}$, respectivamente, sendo as variáveis do gênero feminino significativamente menores que as do masculino. Os incisivos superiores e inferiores apresentaram-se mais inclinados que as médias da literatura, ou seja, $115,2^{\circ} \pm 5,5^{\circ}$ para o $1 . P P$ e $93,9^{\circ} \pm 5,7^{\circ}$ para o IMPA.
\end{abstract}

Palavras-chave: Cefalometria. Má oclusão. Análise facial.

\section{INTRODUÇÃO}

Favorecido pela normalidade das relações esqueléticas estabelecidas geneticamente e perpetuadas pelo crescimento, o paciente Padrão I foi definido por Capelozza Filho como o indivíduo normal com má oclusão, denominado anteriormen- te por Andrews como o portador de má oclusão normal. Contrariamente aos indivíduos Padrão II, Padrão III, Padrão Face Longa e Padrão Face Curta, os pacientes objeto de estudo desse artigo não apresentam discrepância esquelética, seja ela sagital ou vertical que, geneticamente determinadas,

\footnotetext{
Especialista em Ortodontia pela PROFIS-USP-Bauru; Mestre em Ortodontia pela Universidade Metodista de São Paulo; Professora Assistente do Departamento de Ortodontia de Universidade Metodista de São Paulo.

* Professor Doutor da Faculdade de Odontologia da Universidade de São Paulo, USP-Bauru e membro do setor de Ortodontia do HRAC da Universidade de São Paulo, USP-Bauru.

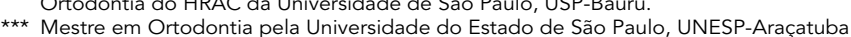

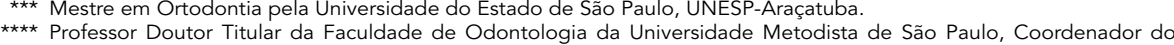
Programa de Pós-Graduação em Odontologia da Universidade de São Paulo.
} 
maculam de forma característica o posicionamento dentário. Assim, a má oclusão nos indivíduos Padrão I é decorrente de desarranjo dentário, geralmente relacionado a fatores etiológicos ambientais, exceto nos paciente com discrepâncias dente-osso positiva ou negativa. Ou seja, nos pacientes Padrão I "o erro dentário é primário, ou a essência da doença” ${ }^{2}$, por isso mesmo inespecífico e dificilmente identificado pela análise facial do paciente.

O diagnóstico do Padrão Facial é realizado por meio da análise morfológica da face, nas avaliações de frente e perfil. O paciente Padrão I é aquele no qual se reconhece o equilíbrio, e não necessariamente a beleza, nas visões frontal e lateral.

$\mathrm{Na}$ análise morfológica frontal, os portadores de Padrão I apresentam simetria aparente, distância intercanto medial dos olhos similar à largura do nariz, distância interpupilar similar à largura da comissura bucal, proporção entre os terços faciais, altura do lábio superior equivalente à metade da altura do lábio inferior, volume proporcional de vermelhão dos lábios e selamento labial passivo, o qual pode estar comprometido nos pacientes acometidos por protrusão da arcada dentária superior.

$\mathrm{Na}$ avaliação do perfil, a normalidade é caracterizada por um grau moderado de convexidade. A expressão da maxila na face é identificada pela presença da projeção zigomática e depressão infra-orbitária, que podem ser verificadas também na visão frontal. A linha de implantação do nariz, normalmente paralela a uma vertical verdadeira, denota adequada posição maxilar. O sulco naso-geniano com leve inclinação posterior completa a avaliação do equilíbrio maxilar. O ângulo nasolabial avalia a relação da base nasal em relação ao lábio superior, cuja posição é fortemente determinada pela inclinação dos incisivos superiores. Portanto, esse ângulo pode estar adequado, aberto ou fechado nos pacientes Padrão I, como consequiência da posição dos dentes anteriores superiores, independente do bom posicionamento maxilar, sempre observado nestes pacientes.

$\mathrm{O}$ equilíbrio mandibular, tamanho, forma e posição, pode ser verificado na avaliação do perfil por meio da linha queixo-pescoço. Ela deve ter um tamanho normal e tende ao paralelismo com o Plano de Camper. Esse paralelismo contribui para um ângulo adequado entre as linhas do queixo e do pescoço. Além disso, espera-se um ângulo mentolabial agradável esteticamente e construído com igual participação do lábio e do mento.

Presume-se que as medidas cefalométricas destes pacientes estejam dentro dos limites definidos como normais. Isso não significa necessariamente que esses valores sejam similares à média, pois esta, ao contrário do correntemente adotado na interpretação cefalométrica, representa apenas um valor médio de medidas obtidas a partir de uma população normal investigada.

Vários estudos cefalométricos já foram realizados com o intuito de definir as medidas dentoesqueléticas de indivíduos sem discrepâncias esqueléticas sagitais ou verticais. Entretanto, a maioria destes trabalhos teve como método de seleção a oclusão normal, partindo da premissa de que os pacientes portadores de oclusão normal teriam também adequadas relações esqueléticas. Esse método de seleção exclui, entretanto, inúmeros indivíduos Padrão I, ou seja, equilibrados esqueleticamente, mas portadores das mais diversas más oclusões, decorrentes, principalmente, de discrepâncias dentárias ou fatores ambientais.

No paciente Padrão I, como anteriormente dito, a má oclusão é primária, ou a essência da doença, portanto esses pacientes podem apresentar qualquer erro no posicionamento dentário, seja transversal, ântero-posterior ou vertical, sem envolvimento esquelético, e normalmente, não identificados pela análise facial. Exceção freqüente a essa observação é o desequilibrio labial ântero-posterior causado pela protrusão dentária superior.

A introdução do conceito de Padrão demanda o estudo de cada um deles para o estabelecimento de suas características faciais, base para o diagnóstico, oclusais e cefalométricas. Ainda sem usar esta nomenclatura, mas utilizando o critério da morfologia facial para a seleção dos pacientes, estudos já foram 
realizados definindo os parâmetros cefalométricos dos pacientes Padrão II e Padrão III. Os trabalhos sobre os Padrões Face Longa e Face Curta estão em desenvolvimento e serão publicados em breve. Por hora, vimos estudar as características cefalométricas de uma amostra de indivíduos Padrão I.

\section{MATERIAL E MÉTODO}

A amostra do presente estudo é constituída por 30 indivíduos selecionados a partir de uma amostra pré-existente composta por 100 brasileiros, adultos, leucodermas, portadores de selamento labial passivo, de ambos os gêneros (50 do gênero masculinos e 50 do gênero feminino), com idade média de 23 anos e 7 meses, variando entre 18 e 36 anos $^{9}$. Nenhum dos pacientes foi submetido previamente a tratamento ortodôntico ou cirurgia plástica facial.

Os 30 indivíduos foram selecionados por terem sido definidos como portadores de Padrão I na avaliação morfológica da face em fotografias de perfil,

\begin{tabular}{|c|}
\hline Medidas Lineares \\
\hline CoA - Comprimento efetivo da maxila \\
\hline CoGn - Comprimento efetivo da mandíbula \\
\hline DifMxMd - Diferença maxilo-mandibular \\
\hline AFAT - Altura facial anterior total \\
\hline AFAI - Altura facial anterior inferior \\
\hline AFAM - Altura facial anterior média \\
\hline AFP - Altura facial posterior \\
\hline AFATperp - Altura facial anterior total perpendicular \\
\hline AFAlperp - Altura facial anterior inferior perpendicular \\
\hline $1-P P$ \\
\hline$\underline{6}-\mathrm{PP}$ \\
\hline 1-PM \\
\hline $6-\mathrm{PM}$ \\
\hline Medidas Angulares \\
\hline SNA \\
\hline SNB \\
\hline ANB \\
\hline AnGon \\
\hline AnPP \\
\hline 1.PP \\
\hline IMPA \\
\hline AI 3IF - Ângulo do terço inferior da face \\
\hline AnPM - Ângulo do plano mandibular \\
\hline NAP \\
\hline Medidas Proporcionais \\
\hline AFAlperp/AFATperp \\
\hline AFAI/AFAT \\
\hline AFAM/AFAI \\
\hline AFP/AFAT \\
\hline
\end{tabular}

independente das relações oclusais. Esses pacientes apresentavam: 1) perfil levemente convexo; 2) terços faciais proporcionais; 3) linha queixo-pescoço paralela ao plano de Camper; 4) sulco mentolabial normal com igual participação do lábio e do mento; 5) projeção zigomática presente, caracterizada pela presença de depressão infra-orbitária e sulco naso-geniano. A decisão de realizar a seleção em fotografias de perfil esteve relacionada ao fato das características morfológicas descritas acima, que definem o Padrão I, serem mais facilmente e objetivamente observadas nesta vista que na frontal, pois as discrepâncias maxilo-mandibulares sagitais e verticais costumam comprometer mais a visão lateral. A porcentagem desses indivíduos também classificados como Padrão I na avaliação da fotografia de frente foi de $93,3 \%$, ou seja, 28 dos 30 selecionados pela análise do perfil.

Esta amostra é composta por 22 indivíduos do gênero feminino e 8 do gênero masculino. A idade média é $22,6 \pm 2,9$ anos para o grupo total, $23,8 \pm$ 3,3 anos para o gênero feminino e $21,6 \pm 1,2$ anos para o masculino.

As telerradiografias cefalométricas laterais dos indivíduos da amostra foram digitalizadas por meio de scanner, sendo as imagens submetidas à análise do programa Radiocef 2.0, seguindo as normas especificadas pelo fabricante ${ }^{12}$. Para a obtenção das medidas lineares, angulares e proporcionais, os pontos cefalométricos, linhas e planos foram demarcados nas imagens escaneadas, por um único observador.

As medidas utilizadas nesse trabalho estão listadas no quadro 1 .

Os resultados foram submetidos à avaliação estatística, calculando-se as médias e desvios-padrões, bem como os valores máximos e mínimos para todas as variáveis mensuradas (Tab. 2). Com o objetivo de detectar as diferenças entre os gêneros, utilizou-se o teste " $t$ " para dados independentes, estabelecendo-se 5\% de significância para todas as comparações realizadas (Tab. 3).

Para a verificação do erro do método, 8 telerradiografias foram aleatoriamente escolhidas, 
cujos pontos foram novamente demarcados, e as mensurações refeitas utilizando a mesma metodologia. Os valores obtidos na primeira e na segunda mensuração foram submetidos ao teste $t$, para o estudo do erro sistemático, e à fórmula de Dahlberg, para a avaliação do erro casual.
De acordo com a estimativa do erro do método (Tab. 1), não foram detectadas diferenças estatisticamente significantes quanto ao erro sistemático, enquanto que os valores do erro casual obtidos encontravam-se dentro de um limite tolerável ${ }^{13}$.

As fotografias faciais de perfil e de frente foram

\begin{tabular}{|c|c|c|c|c|c|c|c|}
\hline Variáveis & Média 1 & DP 1 & Média 2 & DP 2 & $\mathbf{t}$ & $\mathbf{p}$ & Erro \\
\hline Co-A & 96,02 & 6,14 & 96,44 & 6,07 & 1,157 & 0,270 & 0,94 \\
\hline DifMxMd & 31,00 & 5,16 & 30,70 & 4,70 & 0,872 & 0,400 & 0,86 \\
\hline AFAT & 126,75 & 9,26 & 126,62 & 9,24 & 0,393 & 0,701 & 0,81 \\
\hline AFP & 63,46 & 5,44 & 64,21 & 4,72 & 1,227 & 0,243 & 1,60 \\
\hline AFAT perp & 126,58 & 9,07 & 126,40 & 9,04 & 0,518 & 0,614 & 0,85 \\
\hline AFAI perp & 71,16 & 5,79 & 70,95 & 6,06 & 0,627 & 0,542 & 0,82 \\
\hline SNA & 82,53 & 3,35 & 83,00 & 2,57 & 1,358 & 0,199 & 0,92 \\
\hline SNB & 79,86 & 1,92 & 80,23 & 1,72 & 1,890 & 0,083 & 0,55 \\
\hline $1 . P P$ & 113,88 & 8,48 & 113,24 & 8,43 & 0,765 & 0,459 & 2,09 \\
\hline IMPA & 93,03 & 7,10 & 94,00 & 7,23 & 2,079 & 0,060 & 1,33 \\
\hline NAP & 5,51 & 4,88 & 4,96 & 4,47 & 1,387 & 0,191 & 1,05 \\
\hline AFAIperp/AFATperp & 0,56 & 0,02 & 0,56 & 0,02 & 0,365 & 0,721 & 0,01 \\
\hline AFAI/AFAT & 0,57 & 0,02 & 0,57 & 0,02 & 0,693 & 0,502 & 0,01 \\
\hline AFAM/AFAI & 0,77 & 0,06 & 0,78 & 0,06 & 0,391 & 0,703 & 0,01 \\
\hline AFP/AFAT & 0,50 & 0,04 & 0,51 & 0,04 & 1,637 & 0,128 & 0,01 \\
\hline
\end{tabular}

\begin{tabular}{|c|c|c|c|c|c|}
\hline Variáveis & Unid. & Médias(PI) & Mínimo & Máximo & $d p$ \\
\hline Co-Gn & $\mathrm{mm}$ & 124,21 & 108,71 & 138,35 & 8,25 \\
\hline DifMxMd & $\mathrm{mm}$ & 29,01 & 22,48 & 37,88 & 4,05 \\
\hline AFAT & $\mathrm{mm}$ & 123,04 & 107,39 & 142,02 & 8,32 \\
\hline AFAI & $\mathrm{mm}$ & 68,81 & 53,45 & 80,45 & 6,62 \\
\hline AFATperp & $\mathrm{mm}$ & 122,87 & 107,38 & 145,02 & 8,33 \\
\hline AFAlperp & $\mathrm{mm}$ & 67,57 & 53,10 & 78,43 & 6,54 \\
\hline SNA & graus & 82,25 & 74,32 & 90,01 & 2,97 \\
\hline SNB & graus & 79,81 & 73,65 & 86,39 & 2,52 \\
\hline ANB & graus & 2,43 & $-0,73$ & 5,51 & 1,47 \\
\hline IMPA & graus & 93,96 & 84,54 & 107,70 & 5,74 \\
\hline NAP & graus & 3,53 & 0,16 & 9,92 & 2,71 \\
\hline AFAlperp/AFATperp & - & 0,55 & 0,49 & 0,59 & 0,02 \\
\hline AFAI/AFAT & - & 0,56 & 0,50 & 0,60 & 0,02 \\
\hline AFAM/AFAI & - & 0,81 & 0,68 & 1,03 & 0,08 \\
\hline AFP/AFAT & - & 0,51 & 0,45 & 0,59 & 0,03 \\
\hline
\end{tabular}




\begin{tabular}{|c|c|c|c|c|c|c|c|}
\hline Variáveis & Unid. & Médias (F) & $d p$ & Médias (M) & $d p$ & $t$ & $\mathbf{p}$ \\
\hline Co-Gn & $\mathrm{mm}$ & 120,23 & 5,37 & 135,13 & 3,12 & $-7,350$ & $<0,001^{*}$ \\
\hline Co-A & $\mathrm{mm}$ & 92,38 & 3,65 & 102,94 & 2,02 & $-7,702$ & $<0,001^{*}$ \\
\hline DifMxMd & $\mathrm{mm}$ & 27,85 & 3,85 & 32,19 & 2,76 & $-2,910$ & $<0,05^{*}$ \\
\hline AFAT & $\mathrm{mm}$ & 119,14 & 5,43 & 133,78 & 4,31 & $-6,849$ & $<0,001^{*}$ \\
\hline AFAI & $\mathrm{mm}$ & 65,90 & 4,92 & 76,82 & 3,03 & $-5,851$ & $<0,001^{*}$ \\
\hline AFAM & $\mathrm{mm}$ & 54,94 & 3,19 & 58,66 & 2,93 & $-2,879$ & $<0,05^{*}$ \\
\hline AFP & $\mathrm{mm}$ & 61,60 & 3,94 & 65,58 & 5,62 & $-2,182$ & $<0,05^{*}$ \\
\hline AFATperp & $\mathrm{mm}$ & 118,95 & 5,42 & 133,65 & 4,34 & $-6,880$ & $<0,001^{*}$ \\
\hline AFAlperp & $\mathrm{mm}$ & 64,64 & 4,77 & 75,63 & 2,81 & $-6,098$ & $<0,001^{*}$ \\
\hline SNA & graus & 82,09 & 1,90 & 82,68 & 5,04 & $-0,471$ & $>0,05$ \\
\hline SNB & graus & 79,77 & 1,98 & 79,93 & 3,81 & $-0,146$ & $>0,05$ \\
\hline ANB & graus & 2,31 & 1,29 & 2,75 & 1,95 & $-0,702$ & $>0,05$ \\
\hline AnGon & graus & 120,28 & 4,68 & 124,61 & 6,12 & $-2,063$ & $<0,05^{*}$ \\
\hline AnPM & graus & 28,58 & 3,96 & 31,06 & 4,52 & $-1,459$ & $>0,05$ \\
\hline AnPP & graus & 10,18 & 2,91 & 7,42 & 3,55 & 2,167 & $<0,05^{*}$ \\
\hline$\underline{1} . P P$ & graus & 114,81 & 5,89 & 116,45 & 4,63 & $-0,708$ & $>0,05$ \\
\hline IMPA & graus & 93,80 & 6,23 & 94,40 & 4,48 & $-0,245$ & $>0,05$ \\
\hline NAP & graus & 3,21 & 2,77 & 4,43 & 2,46 & $-1,101$ & $>0,05$ \\
\hline AFAlperp/AFATperp & - & 0,54 & 0,02 & 0,56 & 0,01 & $-2,343$ & $<0,05^{*}$ \\
\hline AFAI/AFAT & - & 0,55 & 0,02 & 0,57 & 0,02 & $-1,971$ & $>0,05$ \\
\hline AFAM/AFAI & - & 0,84 & 0,08 & 0,76 & 0,05 & 2,409 & $<0,05^{*}$ \\
\hline AFP/AFAT & - & 0,52 & 0,03 & 0,49 & 0,04 & 2,067 & $<0,05^{*}$ \\
\hline
\end{tabular}

submetidas à Análise Facial Subjetiva ${ }^{9}$, com o objetivo de determinar a porcentagem de indivíduos esteticamente agradáveis, aceitáveis e desagradáveis nessa amostra Padrão I e, portanto, esqueleticamente equilibrada. Os resultados desta análise, obtidos para as duas visões, foram ainda comparados com os achados para a amostra original de 100 pacientes, com o objetivo de verificar se os indivíduos mais equilibrados seriam também mais agradáveis.

Os modelos de oclusão foram também avaliados para determinação da Relação Oclusal Sagital, segundo a classificação de Angle, e os resultados confrontados com o da amostra original. Em hipótese, a possibilidade dos indivíduos Padrão I apresentarem menor porcentagem de más oclusões Classes II e III.

\section{RESULTADOS E DISCUSSÃO}

Os pacientes Padrão I são aqueles que, avaliados pela análise morfológica da face, não apresentam discrepância esquelética vertical ou sagital, e cuja má oclusão, se presente, está restrita ao mal po- sicionamento dentário. Considerando a eficiência da Ortodontia em movimentar dentes, podemos inferir que os prognósticos de correção favorecem os indivíduos portadores deste Padrão.

Quando as fotografias do perfil dessa amostra foram submetidas à análise facial subjetiva ${ }^{9}$ verificou-se que $10 \%$ foram consideradas esteticamente agradáveis, $86,7 \%$ esteticamente aceitáveis e 3,3\% esteticamente desagradáveis. À análise facial subjetiva das fotografias frontais verificou-se $20 \%$ de individuos esteticamente agradáveis, $73,3 \%$ esteticamente aceitáveis e 6,7\% esteticamente desagradáveis.

Ao comparamos estes resultados com os obtidos para a amostra original de 100 indivíduos portadores de selamento labial passivo, que excluía os portadores de discrepâncias verticais e os Padrões II e III mais graves, observamos diferenças importantes. Na análise do perfil, a amostra total apresentou 3\% dos indivíduos esteticamente agradáveis, $89 \%$ esteticamente aceitáveis e $8 \%$ esteticamente desagradáveis, denotando um aumento 
da agradabilidade e uma redução da desagradabilidade no Grupo Padrão I. Deve-se salientar que o Grupo Padrão I faz parte da amostra total, avaliada pelo mesmo grupo de avaliadores.

$\mathrm{Na}$ análise subjetiva frontal, a amostra total foi 12\% esteticamente agradável, $83 \%$ esteticamente aceitável e 5\% esteticamente desagradável, verificando um aumento da agradabilidade na visão frontal, com redução da porcentagem dos indivíduos considerados esteticamente aceitáveis no Grupo Padrão I.

Os resultados obtidos revelam o esperado, pois os pacientes classificados como Padrão I apresentam equilíbrio facial reconhecido principalmente na avaliação do perfil. Padrões II ou III moderados, presentes em vários pacientes da amostra total, podem não macular a face quando avaliada de frente, mas são prontamente identificados pelo aumento ou redução da convexidade do perfil. Por isso, indivíduos Padrão I são mais agradáveis, seja de frente ou perfil, quando comparados a pacientes Padrões II e III. Entretanto, apenas na visão do perfil os avaliadores observaram redução da desagradabilidade nos indivíduos Padrões I.

Observou-se ainda a relação oclusal sagital, segundo a classificação de Angle, e verificou-se que $6,7 \%$ da amostra Padrão I era portadora de oclusão normal, 56,7\% de má oclusão Classe I, 33,3\% de Classe II e 3,3\% de Classe III. Esse achado atesta que os pacientes Padrão I podem ter qualquer tipo de desarranjo dentário, incluindo Classe II e Classe III dentárias, sem qualquer comprometimento esquelético. Para a amostra total observou-se 7\% de pacientes portadores de oclusão normal, $48 \%$ Classe I, 42\% Classe II e 3\% Classe III. A seleção do Grupo Padrão I pela análise morfológica da face excluiu os indivíduos Padrão II e, por conseguinte, reduziu a porcentagem de Classe II e aumentou a porcentagem de pacientes Classe I.

Espera-se que as medidas cefalométricas dos pacientes Padrão I sejam similares às obtidas nos estudos onde foram definidas medidas padrão das várias análises cefalométricas, pois pressupõe-se que os diversos autores selecionaram pacientes esqueleticamente equilibrados para compor as amostras utilizadas na determinação dos valores normativos de referência. Porém, e isso é básico e muito importante, essas medidas não são, necessariamente, similares às médias obtidas, já que média é apenas a média de valores normais obtidos para uma amostra investigada.

Com o objetivo de facilitar a discussão das medidas obtidas nesse trabalho, dividiu-se as várias características pesquisadas em tópicos, de acordo com as grandezas e as áreas craniofaciais.

\section{Padrão de crescimento facial}

O padrão de crescimento facial foi avaliado nesse trabalho por meio do ângulo do plano palatino e do ângulo do plano mandibular. O ângulo goníaco foi também investigado pela sua importância na construção de uma mandíbula normal, resultado de um crescimento adequado que se espera encontrar nos indivíduos Padrão I.

A média obtida para o ângulo do plano mandibular, formado pela confluência das linhas S-N e Go-Gn, foi $29,2^{\circ} \pm 4,2^{\circ}$, variando entre $20,8^{\circ}$ e $33,3^{\circ}$. Observou-se uma tendência deste ângulo estar mais obtuso no gênero masculino $\left(31,0^{\circ} \pm 4,5^{\circ}\right)$ que no gênero feminino $\left(28,6^{\circ} \pm 3,9^{\circ}\right)$, sem significância estatística.

O valor médio de normalidade sugerido pela literatura para este ângulo é $32^{\circ}$, levemente maior que o da amostra, porém dentro de um desvio padrão da média encontrada para a mesma. $O$ fato de $73,3 \%$ da amostra ser do gênero feminino, o qual apresentou uma tendência de valores mais agudos, pode ter contribuído para a diferença encontrada.

O ângulo do plano palatino apresentou uma grande variabilidade na amostra. Apesar da média obtida ter sido $9,4^{\circ} \pm 3,2^{\circ}$, os valores variaram amplamente, entre $2,8^{\circ}$ e $15,8^{\circ}$. Foi verificado ainda dimorfismo sexual, com a média feminina de $10,2^{\circ} \pm 2,9^{\circ}$ e a masculina $7,4^{\circ} \pm 3,5^{\circ}$, não corroborando a tendência vertical do gênero masculino do ângulo do plano mandibular, porém coincidindo com os achados de Martins ${ }^{7}$ para amostra de adultos, brasileiros, leucodermas, portado- 

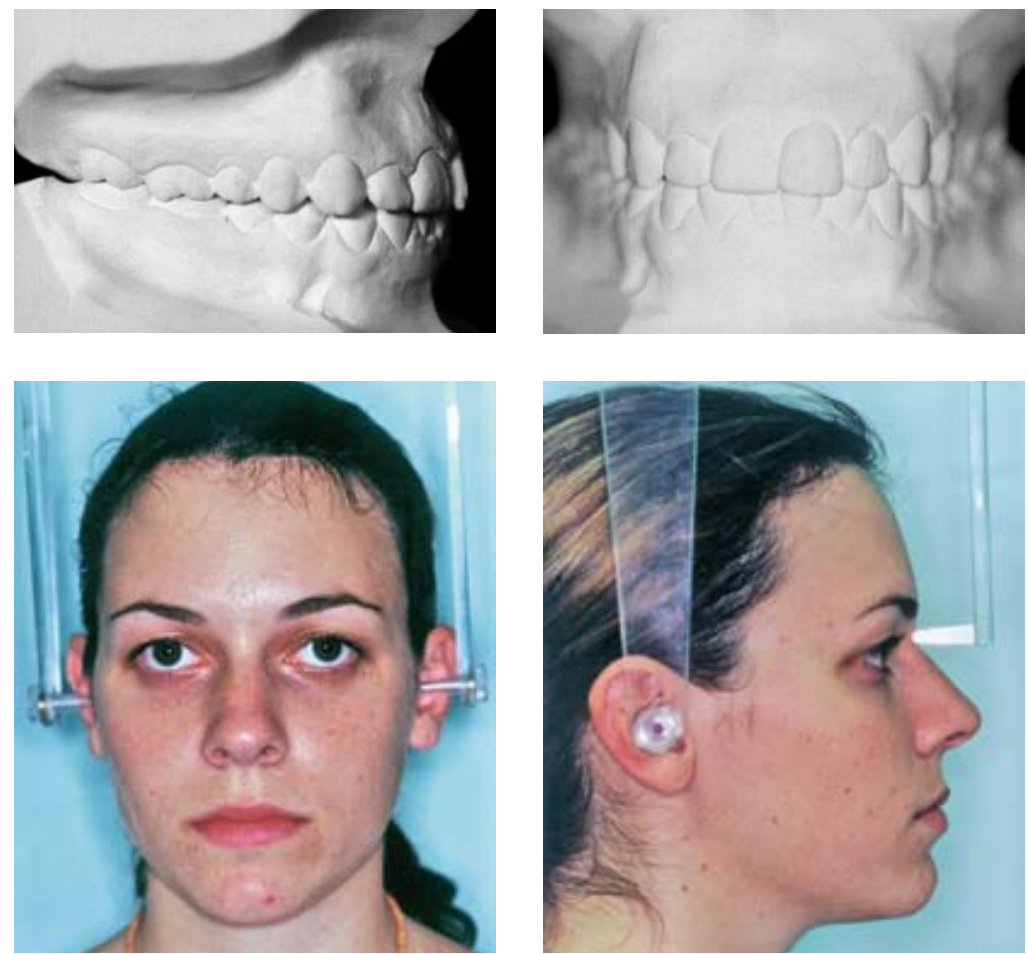

FIGURA 1 - Paciente Padrão I, Classe II, divisão 1, subdivisão esquerda.
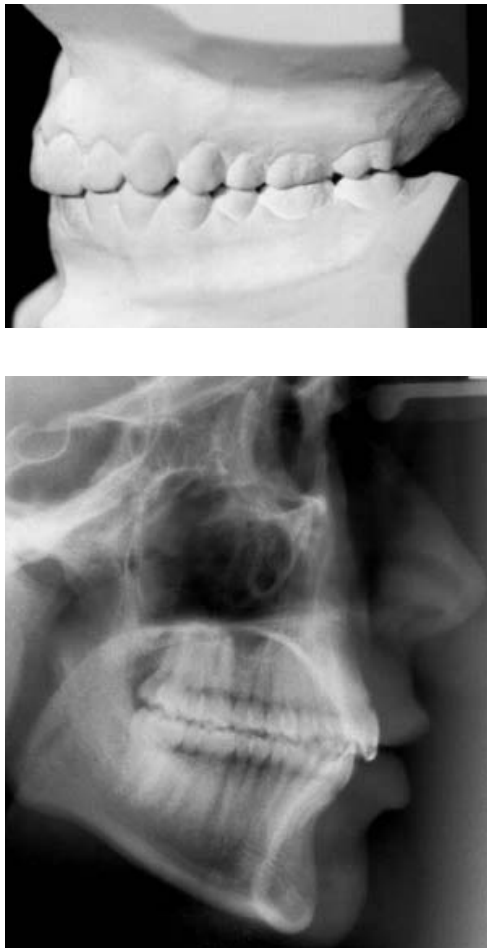

FIGURA 2 - Telerradiografia de perfil de paciente Padrãol. res de oclusão normal $\left(8,6^{\circ} \pm 4,1^{\circ}\right.$ para o gênero masculino e $11,3^{\circ} \pm 3,3^{\circ}$ para o feminino).

O ângulo goníaco está diretamente relacionado à morfologia mandibular. Considerando que a característica principal dos pacientes Padrão I é o equilíbrio facial, espera-se que os mesmos não apresentem alterações importantes na mandíbula, com variações deste ângulo relacionadas ao tipo facial. A média obtida para o mesmo foi $121,4^{\circ} \pm 5,3^{\circ}$, variando entre $109,0^{\circ}$ e $133,0^{\circ}$. Repetindo os resultados obtidos para o ângulo do plano mandibular, observou-se dimorfismo sexual, e os pacientes do gênero feminino apresentaram o tipo facial tendendo a mais horizontal, com redução do ângulo $\left(120,3^{\circ} \pm 4,7^{\circ}\right.$, variando entre $109,0^{\circ}$ e $128,8^{\circ}$ ), enquanto nos pacientes do gênero masculino o valor mínimo obtido foi $115,6^{\circ}$, o máximo foi $133,0^{\circ}$, e a média foi de $124,6^{\circ} \pm 6,1^{\circ}$.

Segundo Martins et al. ${ }^{7}$, a média do ângulo goníaco em adultos, brasileiros, leucodermas, portadores de oclusão normal, medido utilizando-se o ponto articular, ao invés do condílio utilizado neste trabalho, é de $122,4^{\circ} \pm 5,0^{\circ}$ para o gênero masculino e $124,9^{\circ} \pm 7,7^{\circ}$ para o feminino, contrariando a tendência facial mais horizontal do gênero feminino observada neste estudo. Devemos salientar, no entanto, que além da diferença nos pontos utilizados para a obtenção do ângulo, a seleção das duas amostras utilizou parâmetros diferentes, a morfologia facial Padrão I neste grupo e a oclusão normal no grupo utilizado para comparação. Entretanto, observa-se que, apesar das diferenças, as médias obtidas pelo autor encontram-se dentro dos limites definidos pelo desvio padrão da presente amostra, limite este que deve ser invariavelmente considerado em qualquer avaliação de variável cefalométrica.

Os indivíduos dolicofaciais desta amostra devem estar com medidas próximas aos valores máximos, enquanto os valores mínimos devem ter sido obtidos nos braquifaciais. $\mathrm{O}$ desvio padrão deve ser utilizado para a avaliação da normalidade desses indivíduos, 
pois apesar dos mesmos apresentarem medidas em até $10^{\circ}$ diferentes da média, suas faces são equilibradas e devem ser a referência para o estabelecimento da normalidade em pacientes discrepantes, face longa ou face curta. Ou seja, valores distantes da média não indicam, necessariamente, desequilibrio, apenas um arranjo estrutural diferente, obrigando os ortodontistas a utilizarem o desvio padrão de qualquer medida, e não a média, para definir normalidade.

\section{Alturas faciais anteriores, alturas faciais pos- teriores e suas proporções}

Altura facial anterior total e altura facial anterior total perpendicular

Altura facial anterior inferior e altura facial anterior inferior perpendicular

As alturas faciais anteriores, sejam elas total ou inferior, também deveriam ser medidas projetadas na vertical verdadeira, sendo por esse motivo denominadas perpendiculares. Isso teria o objetivo de eliminar uma possivel falha na mensuração dessas dimensões, que poderia ser promovida por uma eventual rotação ântero-superior ou pósteroinferior da mandíbula, alterando a distância entre os pontos sem que os mesmos tenham alterado suas relações verticais. Essas alterações seriam principalmente esperadas nos pacientes com discrepâncias esqueléticas verticais ou sagitais ${ }^{3}$. Desse modo, era esperado que nos pacientes portadores de Padrão I, os valores das grandezas AFAT e AFATperp, e AFAI e AFAIperp seriam similares, devido à ausência de discrepâncias esqueléticas.

A hipótese aventada foi confirmada pelos resultados, descartando a obrigatoriedade do uso das medidas perpendiculares para definição de alturas faciais nos pacientes Padrão I.

A média obtida para a AFAT foi $123,0 \mathrm{~mm} \pm$ $8,3 \mathrm{~mm}$, enquanto o valor obtido para a AFATperp foi $122,8 \mathrm{~mm} \pm 8,3 \mathrm{~mm}$. Para a AFAI, a média obtida foi $68,8 \mathrm{~mm} \pm 6,6 \mathrm{~mm}$, medida próxima aos $67,5 \mathrm{~mm} \pm 6,5 \mathrm{~mm}$, verificados para o AFATperp.

A presença de dimorfismo sexual foi observada, com valores maiores para o gênero masculino (Tab. 2).
Essa diferença era esperada, uma vez que, segundo Nahoum $^{8}$, a altura facial anterior dos indivíduos do gênero masculino é maior que do feminino.

Os valores obtidos para esta amostra estiveram dentro do desvio padrão das médias obtidas por Martins et al. ${ }^{7}$, em adultos, brasileiros, leucodermas, portadores de oclusão normal (AFAI - 69,4mm $\pm 5,9 \mathrm{~mm}$ gênero feminino; $75,1 \mathrm{~mm} \pm$ $6,9 \mathrm{~mm}$ masculino; AFAT - 124,0mm $\pm 5,3 \mathrm{~mm}$ feminino; $130,9 \mathrm{~mm} \pm 7,1 \mathrm{~mm}$ masculino). As diferenças encontradas podem estar relacionadas aos parâmetros utilizados para a seleção dos dois grupos, como já explicado nesse trabalho.

\section{Altura facial anterior média \\ Altura facial posterior}

Da mesma maneira que as outras alturas faciais anteriores, a altura facial anterior média foi significativamente maior nos indivíduos do gênero masculino que do feminino. A média obtida para a amostra total foi $55,9 \mathrm{~mm} \pm 3,5 \mathrm{~mm}$, com o mínimo de $47,7 \mathrm{~mm}$ e o máximo de $64,1 \mathrm{~mm}$.

A altura facial anterior média contribui secundariamente para a determinação do Padrão Facial. Essa afirmação foi confirmada por $\operatorname{Cardoso}^{4}$ que não observou diferenças estatisticamente significantes entre as medidas dessa altura, comparando-se uma amostra Padrão Face Longa com outra Padrão I.

A altura facial posterior também foi significativamente menor no gênero feminino $(61,6 \mathrm{~mm} \pm$ $3,9 \mathrm{~mm}$ para o gênero feminino e $65,6 \mathrm{~mm} \pm 5,6 \mathrm{~mm}$ para o masculino). A média obtida para a amostra total foi $62,6 \mathrm{~mm} \pm 4,7 \mathrm{~mm}$.

A altura facial posterior é também um fator menos significante na determinação do Padrão Facial, podendo estar normal ou reduzida em pacientes $\mathrm{Pa}$ drão Face Longa ${ }^{4}$. A menor dimensão dessa altura contribuiria para gerar uma face longa mais severa.

\section{Proporção entre as alturas faciais}

Para a estética e o equilíbrio, tão importante quanto as grandezas cefalométricas são as proporções entre as várias dimensões da face. A estética é 
afetada, principalmente, por desproporções entre os componentes faciais.

Segundo Suguino et al. ${ }^{11}$, a face equilibrada deve apresentar terços faciais proporcionais. $\mathrm{O}$ estudo das proporções entre as alturas faciais anteriores inferiores e totais (AFAI/AFAT; AFAIperp/AFATperp) revelaram que, nos pacientes Padrão I, a altura facial inferior é, aproximadamente, $50 \%$ da altura facial anterior total (AFAI+AFAM), confirmando a tendência ao equilíbrio esperado para estes pacientes.

A proporção AFAI/AFAT apresentou média de $0,56 \pm 0,02$, variando entre 0,5 e 0,6 , não sendo observada diferença significativa entre os gêneros. Para a proporção AFAIperp/AFATperp a média obtida foi $0,55 \pm 0,02$, com valores máximos e mínimos de 0,59 e 0,49 , respectivamente.

A média verificada para a proporção entre as alturas faciais anteriores média e inferior foi $0,82 \pm 0,08$, variando entre 0,68 e 1,03. Esperase que as faces com valores mais próximos de 1 sejam, supostamente, mais equilibradas. A comparação entre os gêneros mostrou diferenças significativas. Faces femininas com média de $0,84 \pm 0,08$, enquanto as masculinas apresentaram uma tendência do terço inferior apresentar-se maior que o médio, reduzindo a média para $0,76 \pm 0,05$.

A altura facial posterior tendeu a apresentar metade da dimensão da altura facial anterior total nessas faces morfologicamente equilibradas. A média obtida para esta proporção foi $0,51 \pm 0,03$, com dimorfismo de gênero, devido à maior altura anterior total no gênero masculino, às expensas da altura facial anterior inferior.

Segundo Wuerper ${ }^{14}$, Herzberg 5 e Brons ${ }^{1}$, seguindo um consenso da literatura, a estética agradável estaria associada à harmonia e ao equilíbrio das partes constituintes do perfil facial. Para esta amostra Padrão I, portanto selecionada por apresentar equilibrio estrutural, que apresenta $96,7 \%$ de pacientes aceitáveis ou agradáveis na avaliação de perfil e 93,3\% na vista frontal, esperava-se resultados médios próximos aos indicativos de equilíbrio. Essa hipótese foi confirmada, entre- tanto, vale ressaltar a grande variabilidade das medidas obtidas entre os valores mínimo e máximo. Podemos pressupor que, apesar de todos os indivíduos desta amostra apresentarem equilibrio facial e, portanto, esquelético, a presença de faces aceitáveis e agradáveis pode decorrer, além de outros fatores, dessa variação. Indivíduos esteticamente agradáveis teriam as medidas mais próximas aos valores padrão, médios, enquanto os esteticamente aceitáveis e desagradáveis, teriam valores progressivamente mais coincidentes com os extremos encontrados. Estudos posteriores, correlacionando agradabilidade facial com medidas cefalométricas podem confirmar a tendência verificada.

\section{Relação maxilo-mandibular e os comprimen- tos efetivos da maxila e da mandíbula}

Conforme esperado, os valores divulgados por Riedel ${ }^{10}$ e consolidados pela literatura mundial e nacional para serem utilizados como média padrão das variáveis SNA, SNB e ANB coincidem com os obtidos para esta amostra de pacientes Padrão I, denotando um bom posicionamento da maxila e da mandíbula em relação à base do crânio e entre si.

A média para o ângulo SNA foi $82,2^{\circ} \pm 2,9^{\circ}$, variando entre $74,3^{\circ}$ e $90^{\circ}$. O ângulo $\mathrm{SNB}$, por sua vez, variou entre $73,6^{\circ}$ e $86,3^{\circ}$, com média de $79,8^{\circ} \pm 2,5^{\circ}$, resultando em um ANB cujo valor médio foi $2,4^{\circ} \pm 1,4^{\circ}$. Não foi observado dimorfismo sexual para nenhuma das variáveis acima.

Diante da variabilidade observada entre os valores máximo e mínimo, de $15,7^{\circ}$ para o $\mathrm{SNA}, 12,7^{\circ}$ para o $\mathrm{SNB}$ e $6,44^{\circ}$ para o $\mathrm{ANB}$, podemos especular, novamente, que os indivíduos mais agradáveis esteticamente, principalmente na avaliação do perfil, mais impressionado por variações nessas medidas, seriam aqueles cujas medidas, principalmente do ANB, se aproximariam dos valores médios, enquanto os classificados como aceitáveis ou desagradáveis estariam mais próximos dos extremos, apresentando perfis mais côncavos ou convexos.

Diante da normalidade da amostra, a variação do ANB pode estar associada ao tipo facial do indi- 
víduo. Os indivíduos dolicofaciais desse grupo, provavelmente, apresentam seu valor de ANB próximo ao máximo obtido, pois apresentam, caracteristicamente, maior convexidade facial que os mesofaciais. No extremo oposto estariam os braquifaciais, indivíduos com menores incrementos de crescimento vertical e, portanto, perfil menos convexo.

$\mathrm{O}$ adequado posicionamento sagital das bases ósseas, fundamental para a seleção destes indivíduos Padrão I, segundo o critério morfológico utilizado, e consubstanciado pala análise cefalométrica, permite considerar os valores obtidos para os comprimentos efetivos da maxila e mandíbula como os ideais para este Grupo de brasileiros, adultos, leucodermas, portadores de equilíbrio esquelético.

Para o comprimento efetivo da maxila, a média obtida foi $95,2 \mathrm{~mm} \pm 5,7 \mathrm{~mm}$, variando entre $84,5 \mathrm{~mm}$ e $105,4 \mathrm{~mm}$. Para o comprimento efetivo da mandíbula, a média foi $124,2 \mathrm{~mm} \pm 8,2 \mathrm{~mm}$, sendo o valor mínimo de $108,7 \mathrm{~mm}$ e o máximo $138,3 \mathrm{~mm}$. A diferença maxilo-mandibular apresentou média de $29,0 \mathrm{~mm} \pm 4,0 \mathrm{~mm}$. Como todas as outras medidas lineares, essas variáveis apresentaram dimorfismo sexual com as medidas masculinas significantemente maiores que as femininas.

\section{Relações dentárias com as bases apicais}

Em pacientes portadores de discrepâncias esqueléticas, a posição dos incisivos deve ser avaliada em relação à sua base óssea, ou seja, o ângulo entre o incisivo superior e o plano palatino e o incisivo inferior e o plano mandibular. Estas medidas evitam que erros sagitais nas bases ósseas comprometam a avaliação das posições dentárias.

Considerando que uma das aplicações dos valores obtidos nesse trabalho é fornecer uma média de normalidade para adultos, brasileiros, leucodermas, permitindo a quantificação de erros cefalométricos em pacientes desequilibrados, optamos por utilizar as medidas descritas acima.

A média obtida para o ângulo 1.PP foi $115,2^{\circ}$ $\pm 5,5^{\circ}$, com o valor mínimo de $105,8^{\circ}$ e máximo de $128,6^{\circ}$. Este valor está acima do padrão de normalidade sugerido pela literatura $\left(110^{\circ}\right)$, demonstrando maior protrusão dessa amostra de brasileiros normais, já observada em estudos que avaliaram a protrusão labial ${ }^{9}$.

A inclinação dos incisivos inferiores também esteve aumentada em relação ao padrão de normalidade. A média obtida foi $93,9^{\circ} \pm 5,7^{\circ}$, variando entre $84,5^{\circ}$ e $107,7^{\circ}$. Esses valores coincidem com os obtidos em uma amostra de crianças brasileiras, leucodermas com faces harmoniosas e oclusão normal. A média para o IMPA neste estudo foi $93^{\circ}$, variando entre $84^{\circ}$ e $105,5^{\circ}$.

Comparando as amostras feminina e masculina, não houve diferença estatisticamente significante para a posição dos incisivos inferiores e superiores.

A maior protrusão observada nos brasileiros equilibrados, conseqüente da miscigenação racial, inviabiliza o uso de medidas obtidas em amostra constituídas por outros grupos raciais como referência de normalidade ou metas terapêuticas. Os valores padrão devem ser ajustados para o normal da nossa população.

A variabilidade parece ser a regra na posição dos incisivos, pois foi verificada nesse estudo, confirmando o achado de Martins ${ }^{6}$, e pode ser explicada pela variação encontrada para as outras medidas, entre elas o ANB, SNA e SNB. Podemos observar que, apesar de todos os indivíduos dessa amostra serem Padrão I, o valor mínimo de ANB encontrado foi -0,73 e o máximo 5,51, característicos de perfis côncavos e convexos, respectivamente. A variação na posição dos incisivos compensa a discrepância maxilo-mandibular em faces equilibradas funcionalmente, contribuindo para que estes pacientes sejam reconhecidos como Padrão I na fotografia de perfil. Para os pacientes com ANB reduzido o equilíbrio está associado ao aumento do 1.PP e redução do IMPA, enquanto para os pacientes de ANB aumentado o 1.PP tende a estar reduzido e o IMPA aumentado. A compensação natural é responsável pelo equilíbrio observado, mascarando as inúmeras variações nas construções esqueletais. 


\section{CONSIDERAÇÕES FINAIS}

Uma das validades dos estudos cefalométricos é quantificar erros de amostras discrepantes comparando-os com valores normativos. Entretanto, devese considerar as características das amostras a partir das quais estas normas foram definidas. A princípio, as mesmas deveriam ser estabelecidas para cada população, devido às características peculiares de cada grupo, decorrentes, principalmente, da miscigenação. Outra consideração é o critério de seleção da amostra padrão. A oclusão normal foi, por muitos anos, considerada o indício de normalidade. Observamos, entretanto, que da mesma forma que faces equilibradas, denominadas Padrão I, podem apresentar qualquer tipo de má oclusão, a oclusão normal pode ser observada em faces Padrões II, III, Face Longa ou Face Curta com discrepâncias moderadas, passíveis de compensações dentárias naturais ou ortodônticas $^{2}$. Portanto, a característica oclusal não deve ser a referência para a seleção de faces equilibradas, selecionadas, então, a partir da avaliação morfológica da face nas visões frontal e lateral.

Portanto, o estudo cefalométrico das faces Padrão I fornece os padrões de normalidade para comparação com grupos discrepantes dessa mesma população, permitindo quantificações mais realistas dos erros apresentados e oferecendo referências para correções na direção do normal do grupo ao qual o paciente pertence. Os desvios-padrão devem ser considerados, pois os indivíduos podem se encontrar nos extremos dos mesmos e ainda apresentarem uma face normal. Para os casos cirúrgicos, por exemplo, planeja-se a correção da face de um indíviduo Padrão Face Longa considerando como comparativo as característica do Padrão I dolicofacial. Desse modo, admite-se portanto, perfil mais convexo, alturas faciais anteriores aumentadas e incisivos mais verticalizados. Para o Padrão Face Curta o resultado almejado é o Padrão I braquifacial, com medidas cefalométricas situadas no extremo oposto do caso anteriormente citado.

O presente estudo, propõe, portanto, um conjunto de medidas a serem utilizadas como referência para a comparação com outras amostras brasileiras portadoras de discrepâncias, considerando a média e o desvio padrão de cada variável estudada.

\title{
Cephalometric characteristics assessment of Pattern I
}

\begin{abstract}
The aim of this study was to determine cephalometric characteristics of Pattern I patients, to establish averages, and mainly, standard deviation references to be used comparatively with values of skeletally compromised cases. The sample was comprised by 30 white brazilian adults selected by morphologic facial analysis in lateral photographs. In the study of facial growth pattern, the palatine plane angle was $9,4^{\circ} \pm 3,2^{\circ}$, while goniac angle was $121,4^{\circ} \pm$ $5,3^{\circ}$, both with sexual dimorphism. For the mandibular plane angle, the value was $29,2^{\circ} \pm 4,2^{\circ}$, without sexual dimorphism. The average values for total, lower, medium and posterior facial height were, respectively, $123,0 \mathrm{~mm} \pm$ $8,3 \mathrm{~mm}, 68,8 \mathrm{~mm} \pm 6,6 \mathrm{~mm}, 55,9 \mathrm{~mm} \pm 3,5 \mathrm{~mm}$ e $62,6 \mathrm{~mm} \pm 4,7 \mathrm{~mm}$. For all those measures, feminine values were smaller then masculine ones. Cephalometric position of maxillary and mandibular bones confirmed the skeletal balance of the sample. SNA was $82,2^{\circ} \pm 2,9^{\circ}$, SNB was $79,8^{\circ} \pm 2,5^{\circ}$ and ANB was $2,4^{\circ} \pm 1,4^{\circ}$, without sexual dimorphism. Maxillary length was $95,2 \mathrm{~mm} \pm 5,7 \mathrm{~mm}$, while mandibular length was $124,2 \mathrm{~mm} \pm 8,2 \mathrm{~mm}$, with sexual dimorphism. Upper and lower incisors were more protruded them those of literature samples. Upper incisors were studied by 1.PP angle, and the average was $115,2^{\circ} \pm 5,5^{\circ}$, while the value for IMPA was $93,9^{\circ} \pm 5,7^{\circ}$.
\end{abstract}

Key words: Cephalometry. Malocclusion. Facial analysis. 


\section{REFERÊNCIAS}

1. BRONS, R. Facial harmony: standards for orthognathic surgery and orthodontics. London: Quintessence, 1998. p. 166.

2. CAPELOZZA FILHO, L. Diagnóstico em Ortodontia. Maringá: Dental Press Editora, 2004

3. CAPELOZZA FILHO, L.; SOUZA, S. L. M. C.; CAVASSAN, A. O.; OZAWA, T. O. A altura facial anterior inferior na Classe II, divisão primeira por deficiência mandibular. R Dental Press Ortodon Ortop Maxilar, Maringá, v. 9, n. 6, p. 39-47, nov./dez. 2004.

4. CARDOSO, M. A. Estudo das características cefalométricas do padrão face longa. 2003. 163 f. Dissertação (Mestrado em Ortodontia) - Faculdade de Odontologia, Universidade Estadual Paulista, Araçatuba, 2003.

5. HERZBERG, B. L. Facial esthetics in relation to orthodontic treatment. Angle Orthod, Appleton, v. 22, no. 1, p. 3-22, Jan 1952

6. MARTINS, D. R. Estudo comparativo dos valores cefalométricos das análises de Downs e Tweed, com os de adolescentes brasileiros leucodermas, de origem mediterrânea. Ortodontia, São Paulo, v.14, n. 2, p.101-116, maio 1981

7. MARTINS, D. R. et al. Atlas de crescimento crânio-facial. 1. ed. São Paulo: Ed. Santos, 1998.
8. NAHOUM, H. I. Vertical proportions and the palatal plane in anterior open-bite. Am J Orthod, St. Louis, v. 59, p. 273-282, 1971.

9. REIS, S. A. B. Análise facial numérica e subjetiva do perfil e análise da relação oclusal sagital em brasileiros, adultos, leucodermas, não tratados ortodonticamente. 2001. 271f. Dissertação (Mestrado em Ortodontia) - Faculdade de Odontologia, Universidade Metodista de São Paulo, São Bernardo do Campo, 2001.

10. RIEDEL, R. A. The relation of maxillary structures to cranium in malocclusion and in normal occlusion. Angle Orthod, Appleton, v. 22, no. 3, p.142-145, July 1952

11. SUGUINO, R. et al. Análise facial. R Dental Press Ortodon Ortop Maxilar, Maringá, v.1, n.1, p. 86-107, set./out. 1996.

12. VASCONCELOS, M. H. F. Avaliação de um programa de traçado cefalométrico. 2000. $178 \mathrm{f}$. Tese (Doutorado em Ortodontia) - Faculdade de Odontologia, Universidade de São Paulo, Bauru, 2000.

13. WAH, P. L. Y.; COOKE, M. S.; HAGG, U. Comparative cephalometric errors for orthodontic and surgical patients. Int J Adult Orthodon Orthognath Surg, Chicago, v. 10, p. 119-226, 1995

14. WUERPEL, E. H. Ideals and idealism. Angle Orthod, Appleton, v. 1, p.14-31, 1931.

Endereço para correspondência

Sílvia A. Braga Reis

Rua Timbiras, 1560 - Conj. 308

Lourdes

Belo Horizonte/MG

CEP: 30140-061

E-mail: silvia@ortoadultos.com.br 